\title{
Magnetic Properties of Pure Galvinoxyl under Pressure. Suppression of the Structural Change and Observation of the Magnetic Ordering
}

\author{
Y. Hosokoshi, M. Mito, ${ }^{*}$ M. Tamura, ${ }^{\dagger}$ K. Takeda, ${ }^{*}$ K. Inoue, and M. Kinoshita ${ }^{\ddagger}$ \\ Institute for Molecular Science, Myodaiji, Okazaki 444, Japan \\ * Kyushu University, Hakozaki, Fukuoka 812-81, Japan \\ † Toho University, Miyama, Funabashi, Chiba 274, Japan \\ $\ddagger$ Science University of Tokyo in Yamaguchi, Onoda, Yamaguchi 756, Japan
}

\begin{abstract}
We have developed a high-pressure clamp cell made of $\mathrm{Cu}-\mathrm{Ti}$ alloy, which can be equipped to a Quantum Design MPMS SQUID magnetometer. The precise magnetic measurements is possible by using this clamp cell. We have discovered that the structural change of organic radical, galvinoxyl is suppressed by pressurization. Magnetic and thermal properties under pressure have been examined. This compound under $7 \mathrm{kbar}$ is a quasione-dimensional Heisenberg ferromagnet with $2 J / k_{\mathrm{B}} \approx 20 \mathrm{~K}$. Interchain interaction is antiferromagnetic and a three-dimensional antiferromagnetic phase transition is observed at $0.72 \mathrm{~K}$.

[magnetism, heat capacity, pressure clamp cell, organic radical, structural change]
\end{abstract}

\section{Introduction}

Organic radicals are 'soft' magnetic materials and physical properties under pressure is attractive and undeveloped subject. We have developed a high pressure clamp cell, which can be equipped to a Quantum Design MPMS SQUID magnetometer and have studied the magnetic properties of organic radical, galvinoxyl.[1] Galvinoxyl is known to possess a large positive Weiss constant of $11 \mathrm{~K}$, but undergoes a phase transition to a diamagnetic state at $85 \mathrm{~K}$.[2] The heat capacity measurements revealed that the phase transition is a firstorder one with a large entropy change.[3] We have discovered that this structural change can be suppressed by pressurization. The mechanism of the pressure effect is discussed from the pressure dependent behaviour. Moreover, we have examined the magnetic and thermal properties under pressure down to $0.5 \mathrm{~K}$. Quasi-one-dimensional nature is confirmed and antiferromagnetic ordering was observed at $0.72 \mathrm{~K}$.

\section{Experimental}

The static susceptibility and magnetization under pressure was measured with the temperature range of $1.8-300 \mathrm{~K}$ using a Quantum Design MPMS SQUID magnetometer. We have

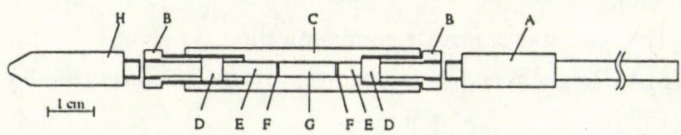

Fig.1. Cross-sectional view of the pressure clamp cell. A: Dummy cell (connected to a sample rod), B: Locking nut, C: Cylinder, D: Backing plate $\left(\mathrm{ZrO}_{2}\right.$, KYOCERA, Z703N), E: Piston $\left(\mathrm{ZrO}_{2}\right)$, F: Cu seal, G: Teflon bucket, $\mathrm{H}$ : Dummy cell.

developed a high pressure clamp cell, which is made of $\mathrm{Cu}-\mathrm{Ti}$ alloy (YAMAHA, YCuTM). This alloy contains no ferromagnetic impurities, and is weakly paramagnetic in the whole temperature range. $\left(\chi_{\mathrm{g}} \approx 2 \times 10^{-9} \mathrm{emu} \mathrm{g}^{-1}\right.$ at $\left.300 \mathrm{~K}\right)$. A cross-sectional view of the cell is given in Fig.1. The outer and inner diameters of the cylinder are 8.5 and $3.0 \mathrm{~mm}$, re spectively. The sample is mounted in a teflon bucket with a inner diameter of $2.4 \mathrm{~mm}$ and a length of $10 \mathrm{~mm}$. The cell enables an internal pressure to be maintained up to about 7 kbar. As a pressure-transmitting medium, a fluorine oil (Montefluos, H-VAC140/13) was used. Although organic radicals are soluble to many solvents and oils, the fluorine oil is inert to organic radicals. The actual pressure at low temperature was calibrated by the superconducting transition temperature of $\mathrm{Pb}$ according to the well-known relation, $d T_{\mathrm{C}} / d p=-0.0365 \mathrm{~K} \mathrm{kbar}^{-1}$.[4] The relation between the

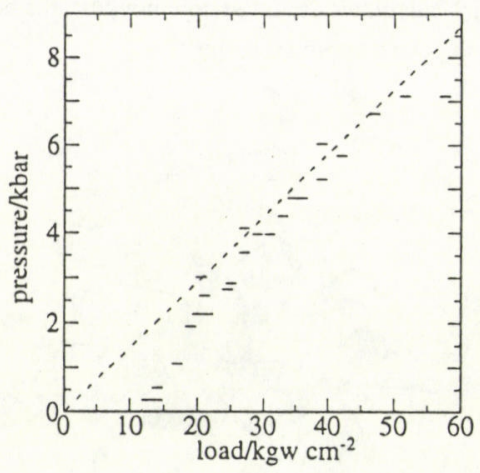

Fig.2. Relation between the pressure at liquid ${ }^{4} \mathrm{He}$ temperature and the load applied at room temperature. The broken line represents the relation simply estimated from load and piston diameter.

pressure at low temperature and the load at room temperature is shown in Fig.2. The difference between the pressure at low temperature and the one applied at room temperature due to the difference in the thermal expansion of the 
transmitting oil and of the cell during the cooling process, is small. In the pressure region of 3-7 kbar, the difference is below ca. 0.3 kbar.

The ac susceptibility and heat capacity under pressure were measured using a ${ }^{3} \mathrm{He}$ cryostat with the $\mathrm{Cu}-\mathrm{Be}$ pressure clamp cell. The detailed procedure is described in the literature.[5]

\section{Results and Discussion}

The temperature dependence of the paramagnetic susceptibility, $\chi_{p}$, under $7 \mathrm{kbar}$ is compared with that at ambient pressure in Fig.3, where $\chi_{\mathrm{p}} T$ is plotted as a function of $T$. It is clear that under $7 \mathrm{kbar}$, the transition is sufficiently

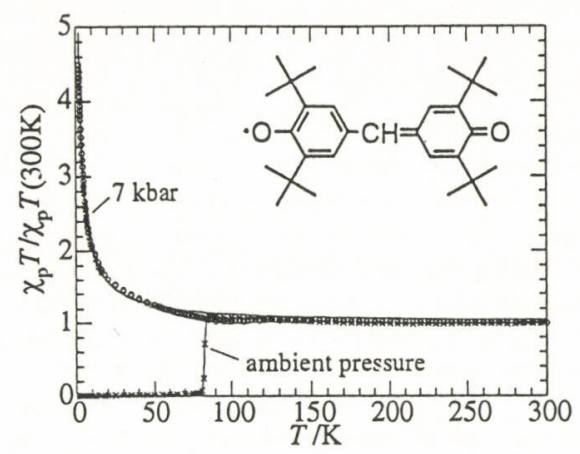

Fig.3. Temperature dependence of $\chi_{\mathrm{p}} T$ of pure galvinoxyl under 7 kbar compared with that at ambient pressure. Solid curve represent the fit by the Heisenberg ferromagnetic chain model with $2 J / k_{\mathrm{B}}=25 \mathrm{~K}$.[6]

suppressed and the ferromagnetic interactions are preserved down to low temperature. The temperature dependence of

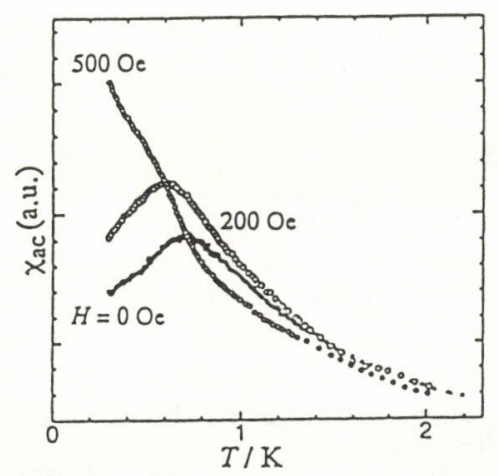

Fig.4. Temperature dependence of ac susceptibility of pure galvinoxyl under 6.4 kbar.

$\chi_{\mathrm{p}} T$ under $7 \mathrm{kbar}$ was analysed on the basis of the Heisenberg ferromagnetic chain model[6] corresponding to the roomtemperature crystal structure.[7] The observed data above $3 \mathrm{~K}$ can be well reproduced with $2 J / k_{\mathrm{B}}=25 \mathrm{~K}$.
We also examined the ground state of this material under $6.4 \mathrm{kbar}$. Figure 4 shows the temperature dependence of the ac susceptibility below $2 \mathrm{~K}$. Under the zero field, the value of the ac susceptibility takes a maximum at around $0.7 \mathrm{~K}$. This maximum temperature decreases with applied fields. Thus, this transition is considered to be an antiferromagnetic one.

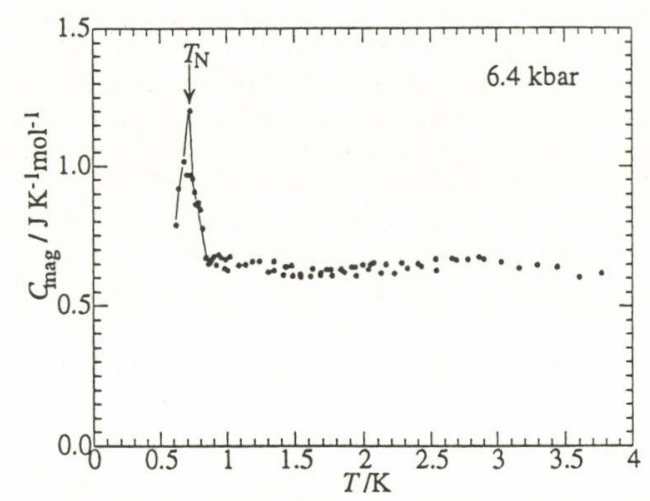

Fig.5. Temperature dependence of heat capacity of pure galvinoxyl under $6.4 \mathrm{kbar}$. Néel temperature $\left(T_{\mathrm{N}}\right)$ is marked by arrow.

We also measured the heat capacity. A $\lambda$-shaped peak was observed at $0.72 \mathrm{~K}$ and the bulk nature of the phase transition was confirmed (Fig.5). We also observed the broad peak at around $10 \mathrm{~K}$. We analysed the field dependence of the broad peak on the basis of the one-dimensional Heisenberg ferromagnetic model and obtained $2 \mathrm{~J} / k_{\mathrm{B}}=18 \mathrm{~K}$.

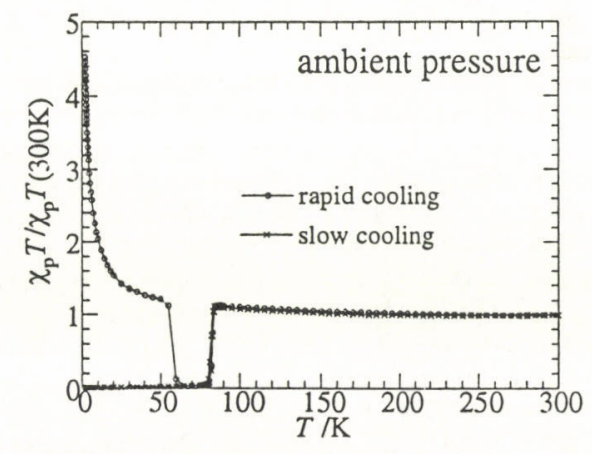

Fig.6. Temperature dependence of $\chi_{\mathrm{p}} T$ of pure galvinoxyl at ambient pressure for the same specimen suffered from a rapid and slow cooling. (see text)

Lastly, we mention the mechanism of the pressure effect. We performed magnetic measurements under several pressures with various cooling rates and observed different behaviours depending on the cooling rates.

Figure 6 shows the temperature dependence of $\chi_{\mathrm{p}} T$ at ambient pressure for the same specimen suffered from a rapid and slow cooling. The measurements in the slow cooling process are shown by crosses. The transition from paramag- 
netic HT (high-temperature) phase to diamagnetic LT (lowtemperature) phase was observed at $82 \mathrm{~K}$ in the cooling process and $84 \mathrm{~K}$ in the warming process. Open circles in Fig.6 represent the measurements in the warming process after the very rapid cooling. Only a very rapid cooling within $5 \mathrm{~min}$. from 300 to $4.2 \mathrm{~K}$ can quench the $\mathrm{HT}$ phase. A moderate cooling with $20 \mathrm{~min}$. from 300 to $4.2 \mathrm{~K}$, cannot maintain the HT phase at all. Below $50 \mathrm{~K}$, since the thermal energy is sufficiently lower than the energy barrier of the transition, the rapid cooled sample can maintain the quenched HT phase as a non-equilibrium state. Above $60 \mathrm{~K}$, the specimen gains enough thermal energy to reach thermal equilibrium, and the rapid and slow cooled sample show the same behaviour.

The temperature dependence of $\chi_{\mathrm{p}} T$ under $2 \mathrm{kbar}$ for the same specimen suffered from a rapid and slow cooling is compared in Fig.7. Open circles represent the measurements in

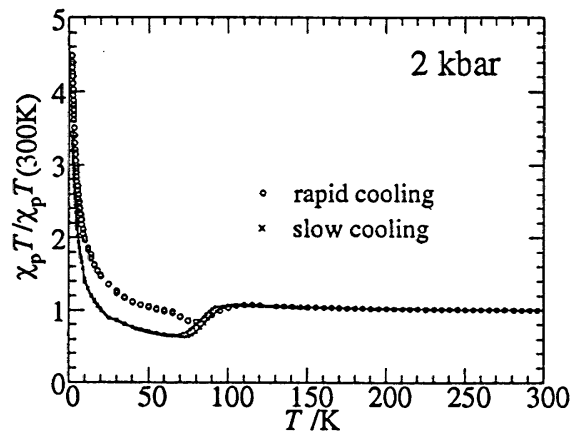

Fig.7. Temperature dependence of $\chi_{\mathrm{p}} T$ of pure galvinoxyl under $2 \mathrm{kbar}$ for the same specimen suffered from a rapid and slow cooling. (see text)

the warming process after the rapid cooling. The measurements in the slow cooling process and the successive warming process are represented by crosses. The cooling rate in the rapid cooling was $10 \mathrm{~min}$. from 300 to $4.2 \mathrm{~K}$ and the one in the slow cooling was $300 \rightarrow 150 \mathrm{~K}, c a .9 \mathrm{~h} ; 150 \rightarrow 100 \mathrm{~K}$, ca. $2.5 \mathrm{~h}$; $100 \rightarrow 50 \mathrm{~K}, \mathrm{ca.} . \mathrm{h}$. By the rapid cooling, the HT phase can be quenched. In the temperature region from 60 to $100 \mathrm{~K}$, the values of $\chi_{\mathrm{p}} T$ decrease but do not fall into zero. Even in the slow cooling process, the values of $\chi_{\mathrm{p}} T$ take finite values below the transition temperature. Insufficient transition occurs between 92 and $68 \mathrm{~K}$ in the slow cooling process, and between 72 and $100 \mathrm{~K}$ in the warming process. The time dependence of the value of $\chi_{\mathrm{p}}$ was checked at $90 \mathrm{~K}$. After 5 days, the change in the $\chi_{\mathrm{p}}$ value was within $0.5 \%$. The measurements after keeping the sample at $100 \mathrm{~K}$ during 3 days gave the same results as that of the slow cooling in Fig.7. The slowcooled sample shows the same $H / T$ dependence of $M / M_{\text {sat }}$ as that of the rapid-cooled sample, although the saturation value is smaller by the factor of 0.75 . The same factor can be estimated from the value of $\chi_{\mathrm{p}} T$ at $1.8 \mathrm{~K}$. It is evident that the paramagnetic reminder in the slow cooling process is $75 \%$ of the HT phase.

It is worth mentioning that the incomplete transition never occur in a first-order phase transition, in other words, the $\begin{array}{ll}\text { (a) at ambient pressure }(p=0) & \text { (b) under pressure }(p)\end{array}$
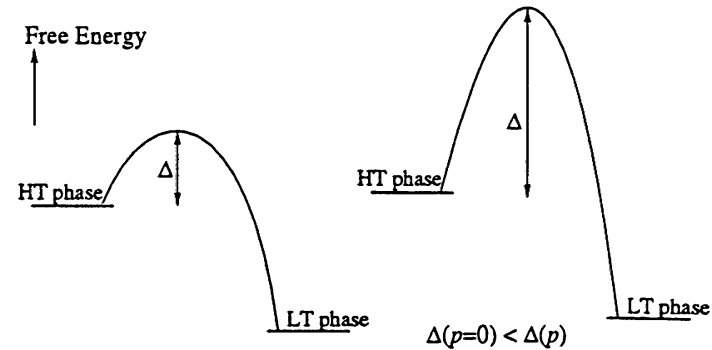

Fig.8. Schematic display of energy diagram of the transition. Energy barrier $(\Delta)$ becomes larger as increasing pressure $(p)$.

incomplete transition is corresponding to the observation of a non-equilibrium state. The thermal equilibrium is related to the minimum free energy but the relaxation time to reach the equilibrium state is affected by the energy barrier of the transition. If the energy barrier is sufficiently higher than the thermal energy, the relaxation time becomes longer and the HT phase can be quenched as a non-equilibrium state.

The observed paramagnetic reminder in the slow cooling under 2 kbar is $75 \%$ of the HT phase quenched as a nonequilibrium state. Since the energy barrier becomes higher under 2 kbar than at ambient pressure, only $25 \%$ of the specimen can surpass the barrier to reach the thermal equilibrium in the experimental time scale.

The amount of the quenched HT phase by the slow cooling increases as increasing pressure. Under $5 \mathrm{kbar}, 81 \%$ of the HT phase can be quenched by the slow cooling. Under $7 \mathrm{kbar}, 100 \%$ of the HT phase can be maintained down to low temperature, although a small anomaly is still detected at around $95 \mathrm{~K}$.

In conclusion, pressurization of pure galvinoxyl causes the enhancement of the energy barrier of the transition. Under $7 \mathrm{kbar}$, the energy barrier of the phase transition is sufficiently high so that the thermal equilibrium cannot be reached at all in the experimental time scale.

\section{Summary}

Low-temperature magnetic and thermal properties of pure galvinoxyl under pressure have been examined. This compound is a quasi-one-dimensional Heisenberg ferromagnet with $2 \mathrm{~J} / k_{\mathrm{B}} \approx 20 \mathrm{~K}$ and undergoes antiferromagnetic ordering at $0.72 \mathrm{~K}$.

\section{References}

[1] Y. Hosokoshi, M. Tamura, and M. Kinoshita, Mol. Cryst. Liq. Cryst., 306, 423 (1997).

[2] K. Mukai, Bull. Chem. Soc. Jpn., 42, 40 (1969).

[3] A. Kosaki, H. Suga, S. Seki, K. Mukai, and Y. Deguchi, Bull. Chem. Soc. Jpn., 42, 1525 (1969).

(4) A. Eiling and J. S. Schilling, J. Phys. F, 11, 623 (1981). [5] K. Takeda, M. Wada, M. Inoue, and T. Haseda, Jpn. J. Appl. Phys., 26,947 (1987).

[6] G. A. Baker Jr. , G. S. Rushbrooke, and H. E. Gilbert, Phys. Rev. A, 135, 1272 (1964).

[7] D. E. Williams, Mol. Phys., 16, 145 (1969). 